This item was submitted to Loughborough's Research Repository by the author.

Items in Figshare are protected by copyright, with all rights reserved, unless otherwise indicated.

\title{
The application of porous media to simulate the upstream effects of gas turbine injector swirl vanes
}

PLEASE CITE THE PUBLISHED VERSION

http://dx.doi.org/10.1016/j.compfluid.2013.03.001

PUBLISHER

(C) Elsevier

VERSION

AM (Accepted Manuscript)

\section{PUBLISHER STATEMENT}

This work is made available according to the conditions of the Creative Commons Attribution-NonCommercialNoDerivatives 4.0 International (CC BY-NC-ND 4.0) licence. Full details of this licence are available at: https://creativecommons.org/licenses/by-nc-nd/4.0/

\section{LICENCE}

CC BY-NC-ND 4.0

\section{REPOSITORY RECORD}

Ford, Christopher L., Jonathan F. Carrotte, and Alastair Duncan Walker. 2015. "The Application of Porous Media to Simulate the Upstream Effects of Gas Turbine Injector Swirl Vanes". figshare. https://hdl.handle.net/2134/19087. 


\title{
The Application of Porous Media to Simulate the Upstream Effects of Gas Turbine Injector Swirl Vanes
}

Ford, C. L., Carrotte J. F. and Walker, A. D.

\begin{abstract}
Numerical simulations are an invaluable means of evaluating design solutions. This is especially true in the initial design phase of a project where several simulations may be required as part of an optimisation study. The design of aircraft gas turbine combustor external aerodynamics frequently calls upon the services of numerical methods to visualise the existing flow field, and develop architectures which improve the performance of the system. Many of these performance improvements are driven by the desire to reduce fuel burn and cut emissions lowering the environmental impact of aviation. The gas turbine combustion chamber is, however, reasonably complex geometrically and requires a high fidelity model to resolve small geometric details. The fuel injector is the most geometrically complex component, requiring around $20 \%$ of the mesh cells of the entire domain. This makes it expensive to model in terms of both requisite computational resource and run time. Most modern aircraft gas turbines utilise swirling flow fields to stabilise the flame front in the combustion liner. The swirl cone is generally generated using fixed angle vane rows within the injector. It is these small features that are responsible for the requisite high mesh cell count. This paper presents a numerical method for replacing the injector swirl vane passages with mathematically porous volumes which replicate the required pressure drop. Modelling using porous media is preferential to modelling the fully featured injector as it allows a significant reduction in the size of the computational domain and number of cells. Additionally the simplification makes the geometry easier to change, scale and re-mesh during development. This in turn allows significant time savings which serve ultimately to expedite the design process. This method has been rigorously tested through a range of approach conditions and flow conditions to ensure that it is robust enough for use in the design process. The loss in accuracy owing to the simplification has been demonstrated to be less than $4.4 \%$, for all tested flow fields. This error is dependent on the flow conditions and is generally much less for passages fed with representative levels of upstream distortion.
\end{abstract}




\section{Nomenclature}

\begin{tabular}{|c|c|}
\hline$A_{1}$ & Geometric inlet area (vane passage) \\
\hline$A_{2}$ & Geometric outlet area (vane passage) \\
\hline$B$ & Blockage ratio \\
\hline$C$ & Total velocity magnitude \\
\hline C & Swirl vane chord \\
\hline$D_{i}$ & Pipe inner diameter \\
\hline$D_{i j}$ & Viscous loss coefficient \\
\hline$D_{o}$ & Pipe outer diameter \\
\hline$E$ & Average velocity magnitude error \\
\hline$f$ & Frequency of distorted profile \\
\hline$h$ & Height of distorted profile \\
\hline$K_{x}$ & Axial Porous loss coefficient \\
\hline$K_{\theta}$ & Tangential Porous loss coefficient \\
\hline$L$ & Porous media axial length \\
\hline$p$ & Static pressure \\
\hline$P$ & Total pressure \\
\hline$q$ & Dynamic pressure \\
\hline$S_{i}$ & Momentum sink scalar \\
\hline$u$ & Axial velocity component \\
\hline$u_{h}$ & Maximum inlet profile velocity \\
\hline$v_{\theta}$ & Tangential velocity component \\
\hline$\gamma_{1}$ & PM/vane leading edge swirl angle \\
\hline$\gamma_{2}$ & Vane exit swirl angle \\
\hline$\gamma_{2}^{\prime}$ & PM exit swirl angle \\
\hline$\theta_{x}$ & Ratio of $K_{\theta}$ to $K_{x}$ \\
\hline$\lambda$ & Total pressure loss coefficient \\
\hline$\mu$ & Fluid dynamic viscosity \\
\hline 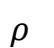 & Fluid density \\
\hline
\end{tabular}


Subscripts

$v$

Referring to vaned geometry

m Referring to Porous media

\section{Superscripts}

Area weighted spatial mean value

Mass weighted spatial mean value 


\subsection{Introduction}

In aero-style gas turbine combustion systems the velocity of the flow issuing from the upstream compressor spool must be reduced and the flow distributed to the various flame tube features. These external aerodynamic processes are typically achieved using a dump diffuser type architecture in which an annular diffuser is followed by an abrupt expansion into a dump cavity (Figure 1). The various flame tube geometrical features then generate the required internal aerodynamic flow fields necessary for combustion to be sustained. For example, vanes within the injector impart tangential momentum to the flow and create a swirl induced recirculation zone for flame stabilisation. Strong downstream and upstream interactions occur between these internal and external aerodynamic flow fields. Authors such as Fishenden and Stevens [1], Carrotte et al. [2] and Walker et al. [3] have shown the significant influence of the pressure field, generated by the flame tube and fuel injectors, on the upstream annular diffuser. Likewise, Barker and Carrotte [4] have shown how non-uniformities presented to the fuel injector, associated with compressor outlet guide vane (OGV) wakes, affect the flow field within the downstream flame tube. In modern low emission lean burn systems, currently under development, a greater proportion of the flow must pass through the fuel injectors which are therefore larger and typically consist of multiple passages $[5,6]$. The increasing size and mass flow requirement of these injectors can potentially enhance the interaction of the fuel injector with the upstream external aerodynamic processes [3]. Additionally, to ensure a uniform air-fuel ratio (AFR) and suitable low emissions performance it is important that a uniform flow is presented to the fuel injectors by the external flow field.

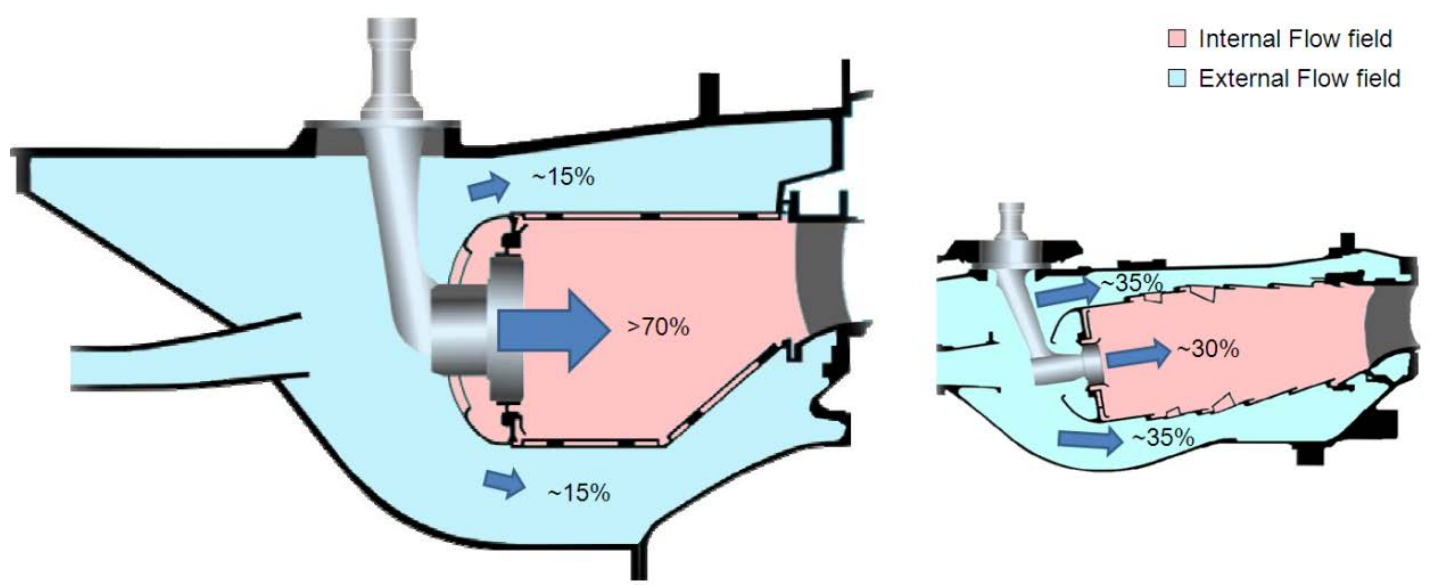

Figure 1: Comparison of Lean Burn (left) and Rich Burn (right) Architectures

Numerical simulations are often be used to help identify injector feed quality and are an intrinsic part of the design process. However, to correctly capture the various upstream and downstream interactions requires the 
external and internal aerodynamic processes to be simulated, see for example Lazik et al. [6]. This computationally expensive, additionally the modelling methods used to simulate the external aerodynamics are less suitable for the internal aerodynamic processes and vice-versa. It is, of course possible to model the external or internal flow field separately. When examining the external flow field only, the fuel injector may be replaced by appropriate exit boundary conditions. This approach was used by Walker [7] to develop a novel combustor pre-diffuser. However, this methodology will not correctly capture the upstream effect of the pressure field associated with the aerodynamic processes undertaken by the flow passing through the injector swirl vanes and into the flame tube and is therefore unlikely to be accurate in the injector near field.

A large injector pressure drop, whilst being detrimental to the engine cycle, would result in an upstream pressure field that causes the flow to redistribute and reduce the magnitude of any spatial variations in the pressure and velocity of the flow presented to the injector. This is a prime example of the interaction which exists between downstream processes and the approaching flow. It is crucial to capture these interactions correctly in order that the flow distribution entering the swirl vanes is correctly simulated. Hence the potential magnitude of any spatial AFR variations in the downstream flow field can be assessed.

This paper describes a numerical methodology which allows the injector swirl vane geometry and downstream flame tube to be replaced with a volume that is mathematically designated porous [8]. In this way the correct gross static pressure field at the leading edge of the injector swirl vanes can be generated. This is effectively achieved by creating a pressure drop that broadly mimics that which would occur within the injector swirl vanes and downstream flame tube. By utilising this approach the need to model the downstream geometry and the associated complexities are avoided. The methodology is easy to employ and since the vane passage is replaced by a prismatic volume it is easy to scale, adjust and re-mesh. All of these advantages result in a shorter simulation time, which in the context of initial design iterations may result in a significant shortening of the design process. 


\subsection{Porous Medium Methodology}

\section{$2.1 \quad$ System Pressure Losses}

Based on a simple 1D analysis, the change in pressure incurred by a stream tube as it passes through a typical gas turbine combustion system is considered (Figure 2). Aerodynamic processes external to the flame tube result in changes to both total and static pressure. Through the diffuser and dump cavity regions a reduction in total pressure is observed which is accompanied, in general, by an increase in static pressure. Upon entering the injector swirl vane passages the static pressure is then reduced, mainly due to flow acceleration (although a small reduction associated with a drop in total pressure will also occur). It is assumed that at exit of the injector the flow undergoes a sudden expansion, with all the kinetic energy being converted into turbulence at some downstream location.

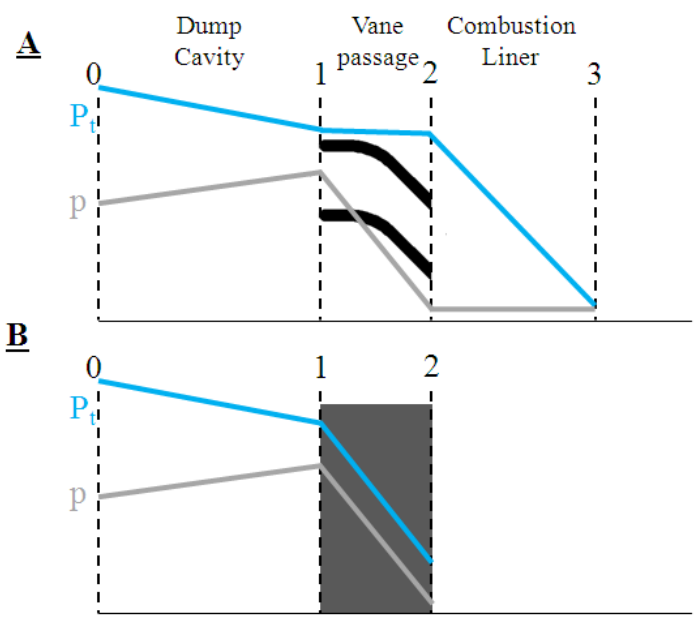

Figure 2: Pressure Variation through the System (A) Fully Featured, (B) With Porous Media

With reference to Figure 2, the total system pressure loss can be expressed in terms of the losses incurred by the flow in the various regions:

$$
\Delta P_{A(0-3)}=\Delta P_{(0-1)}+\Delta P_{(1-2)}+\Delta P_{(2-3)}
$$

Eqn. 1

This may be re-written as:

$$
\Delta P_{A(0-3)}=\Delta P_{(0-1)}+\Delta p_{v}+q_{1}
$$

Eqn. 2

Where $\Delta p_{v}$ is the change in static pressure across the vane passage, and $q_{1}$ is the dynamic head at the swirl vane entry plane. If the vane passage and downstream regions within the combustor (1-3) are replaced by a 
porous medium (Figure 2B) such that the conditions at inlet to the vane passage (1) replicate those that would occur in the complete (vane passage and combustor) system, the losses may be expressed as:

$$
\Delta P_{B(0-2)}=\Delta P_{(0-1)}+\Delta P_{m}
$$

Eqn. 3

The magnitude of the total pressure drop across the porous medium must therefore equal the static pressure drop within the injector vane passages, plus the vane inlet dynamic head:

$$
\Delta P_{m}=\Delta p_{v}+q_{1}
$$

Eqn. 4

\subsection{Injector Swirl Vane Geometry}

The flow within the vane passage, at a plane of constant radius, is shown in Figure 3. At inlet and exit the flow velocities and angles are: $C_{1}, C_{2}, \gamma_{1}$ and $\gamma_{2}$ respectively. If it is assumed that the total pressure loss between stations 1 and 2 is given by $\Delta P_{v}$ then, for incompressible flow:

$$
p_{1}-p_{2}=\Delta p_{v}=\frac{\rho}{2}\left(C_{2}^{2}-C_{1}^{2}\right)+\Delta P_{v}
$$

Eqn. 5

Which may be expressed as:

$$
\Delta p_{v}=\frac{u_{1}^{2} \rho}{2}\left\{\left(\frac{A_{1} \sec \gamma_{2}}{A_{2} B}\right)^{2}-\sec ^{2} \gamma_{1}\right\}+\Delta P_{v}
$$

Eqn. 6

The geometric inlet and exit areas are denoted by $A_{1}$ and $A_{2}$ respectively and $B$ is a blockage ratio which expresses the ratio of effective to geometric area at vane exit, accounting for the finite thickness of the boundary layers etc. In reality the value of B is small and can be typically set to 1.0 (as in the subsequent results that are presented) but it has been included here for completeness.

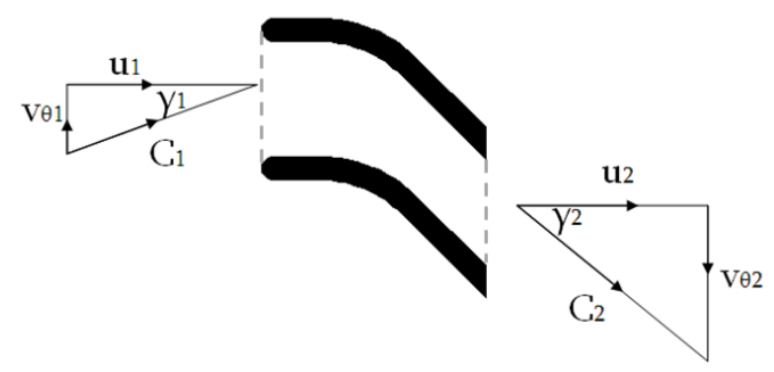

Figure 3: Swirl Vane Schematic 


\section{3}

\section{Derivation of PM Loss Coefficients}

In most CFD codes a volume may be specified as a porous medium (PM) which acts as a user defined momentum sink. This is typically composed of losses associated with viscous and inertial components. Since it is only necessary to replicate a loss value either of these terms can be used. Hence to reduce mathematical complexity the methodology presented here is based on using the inertial losses only. However, this is also thought more appropriate for the fully turbulent flows being considered. In this case, the momentum sink term, $\mathrm{S}$ is given by [8]:

$$
S_{i}=-\frac{1}{2} \sum_{j=1}^{3} K_{j} \rho C v_{j}
$$

Eqn. 7

The porous medium is fully defined by the three coefficient terms: $K_{x}, K_{r}, K_{\theta}$, where the subscripts $x$, $r$ and $\theta$ refer to the axial, radial and tangential directions respectively (relative to an axis defined through the injector centreline). In fuel injector swirl passages there are generally negligible effects associated with the radial velocity component thus it has been assumed that this can be ignored (i.e. $K_{r}=0.0$ ).

Since it is only necessary to replicate a loss it could be assumed that it is only necessary to consider the axial loss coefficient. Reduction to a one dimensional form significantly reduces the mathematical complexity. However, it will be subsequently shown that the one dimensional form is less appropriate for the flows considered and for higher accuracy a two dimensional formulation is necessary. Generally, the two loss components may be in any given ratio, therefore to allow the closure of the equation, the ratio, $\theta_{\mathrm{k}}$ is introduced to relate the tangential to axial coefficients:

$$
\Delta P_{m}=\frac{\rho K_{x}}{2} \int_{0}^{L} C\left(u d x+\theta_{k} v_{\theta} r d \theta\right)
$$

Eqn. 8

Where:

$$
\theta_{k}=\frac{K_{\theta}}{K_{x}}
$$

Now consider the flow through a porous medium as shown by the schematic in Figure 4. Generally, any streamline passing through the medium will experience a total pressure loss as a result of the axial and tangential velocity components. Since the axial velocity must remain constant due to continuity of mass, retardation of tangential velocity will occur. Thus the streamline will curve as it passes through the volume. 
Assuming the inlet flow angle is given by $\gamma_{1}$ and the media outlet angle by $\gamma_{2}^{\prime}$ a relationship for the total pressure loss may be derived as a function of the velocity, media coefficients, and local flow angle, $\gamma(x)$. Assuming that the axial velocity is invariant within the PM and substituting tangential terms for their axial counterparts it is possible to write:

$$
\Delta P_{m}=\frac{\rho K_{x} u_{1}^{2}}{2} \int_{0}^{L}\left\{\left(1-\theta_{k}\right) \sec \gamma+\theta_{k} \sec ^{3} \gamma\right\} d x
$$

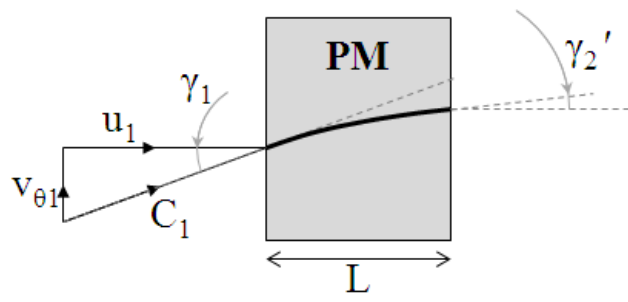

Figure 4: Porous Medium Schematic

Recall that, as shown by Equation 4, the total pressure loss of the porous section should be equal to the static pressure drop across the vanes plus the dynamic pressure at inlet. Combining Equations 6 and 10, and rearranging, an equation for the value of $\mathrm{K}_{\mathrm{x}}$ can be derived, viz:

$$
K_{x}=\frac{k_{0} \sec ^{2} \gamma_{1}}{\int_{0}^{L}\left\{\left(1-\theta_{k}\right) \sec \gamma+\theta_{k} \sec ^{3} \gamma\right\} d x}
$$

Where:

$$
k_{0}=\left(\frac{A_{1} \cos \gamma_{1}}{A_{2} B \cos \gamma_{2}}\right)^{2}+\frac{\Delta P_{v}}{q_{1}}
$$

In the case of pure axial inlet flow $\left(\gamma_{1}=0\right)$, Equation 11 simply reduces to: $K_{x}=\frac{k_{0}}{L}$. However, in the general case an estimation of the behaviour of the flow angle through the PM is required. A good approximation has been found to be given by:

$$
\gamma \approx \gamma_{1} e^{\Gamma_{o} x}
$$

Where: $\Gamma_{\mathrm{o}}$ is a constant given by: 


$$
\Gamma_{o}=\frac{1}{L} \ln \left(\frac{\gamma_{2}^{\prime}}{\gamma_{1}}\right) \mid \gamma_{1} \neq 0
$$

Thus the integrand in the denominator of Equation 11 becomes:

$$
\int_{0}^{L}\left\{\left(1-\theta_{k}\right) \sec \gamma+\theta_{k} \sec ^{3} \gamma\right\} d x \approx \frac{1}{\Gamma_{o}} \int_{\gamma_{1}}^{\gamma_{2}^{\prime}} \frac{\left(1-\theta_{k}\right) \sec \gamma+\theta_{k} \sec ^{3} \gamma}{\gamma} d \gamma
$$

\subsection{Implementation}

Achieving an analytical solution for Equation 15 is problematic and the integration can only be practically achieved numerically. The "gamma approximation" results in a small error in the prediction of K, the magnitude of which will depend on the flow conditions. This is because the assumed gamma profile only ever utilises the inlet and outlet angles and the rate of turning is never correctly captured. However, at small inlet angles (less than around $20^{\circ}$ ) the error in $\mathrm{K}$ is negligibly small (less than $1 \%$ ). The error increases when a higher range of approach angles are experienced but has been shown to be less than 3\% under all tested conditions. The velocity profiles generated with the porous medium employed are not so sensitive to the value of $\mathrm{K}$ and this approximation error is negligible.

With knowledge of the geometric areas of the vane passage and the swirl vane angles it is possible to calculate the approximate value of $\mathrm{K}_{\mathrm{x}}$ required. The approach and exit angles may be solved iteratively, by initially assuming a zero value, i.e. $\gamma_{1}=0$, then correcting the value of $\mathrm{K}_{\mathrm{x}}$ using the mass weighted average approach and PM exit angles, numerically integrating Equation 15 to find a value of the denominator of Equation 11. The iterative procedure required for correct use of the porous medium is not as arduous as it may appear, with solutions tending to converge rapidly after an adjustment in K. Typically only a single iteration is required. For small inlet angles $\left(<5^{\circ}\right)$ the zero inlet angle assumption is usually suitably accurate.

Initial vaned results suggested that the total pressure loss term: $\frac{\Delta P_{v}}{q_{1}}$ (in Equation 12) was typically about 0.6 over a range of inlet conditions and this value was therefore adopted. Furthermore the most accurate value of $\theta_{\mathrm{k}}$ was found to be 1.0, meaning that the axial and tangential loss coefficients were of equal magnitude. A fuller examination of the sensitivity of the K equation to the various terms will be subsequently discussed. 


\subsection{Proof of Concept}

The robustness of the methodology was tested using a simplified model prior to applying it to a more engine representative configuration. The basic model utilised an annular pipe into which a set of swirl vanes could be located or the vanes could be replaced with a porous media. The swirl vanes are typical of those found within modern combustion systems and the duct inlet and exit sections corresponding to approximately 6 and 12 vane chord lengths. Both the inlet and outlet sections had zero wall shear stress to eliminate the influence of any boundary layer growth on the vane flow. When replacing the vanes with a PM the outlet section was unnecessary and the outflow boundary was simply placed at the termination of the PM. Calculations were also undertaken with the PM loss coefficients set to zero. This is equivalent to modelling the injector without any influence of the vane pressure drop and is typical of current practice. Calculation times were reduced by a factor of greater than 100 with the vanes replaced by the PM. (For example, using a single processor on a standard desktop computer simulations times were reduced from more than 24 hours to of order minutes).

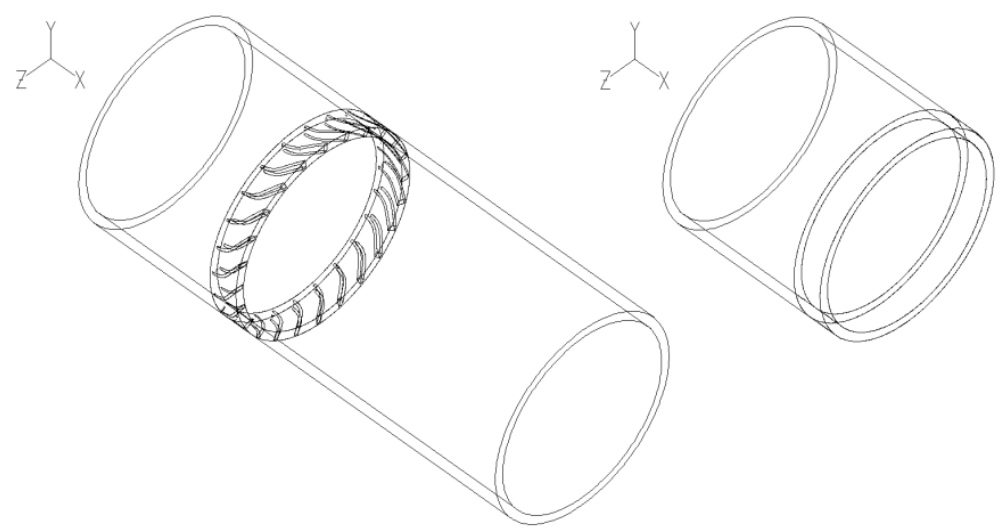

Figure 5: Vaned "Pipe” Geometry and PM Equivalent

In gas turbine combustion systems the fuel injector will, in general, not be uniformly fed. To make the proof of concept calculations more realistic various distorted axial velocity profiles were presented to the vanes/PM. In the first instance the inlet profile was set as a simple square wave with the maxima located at the 3 and 9 o'clock positions consistent with a pronounced pre-diffuser footprint (subsequently dubbed boundary condition 5 - BC05). The distortion was controlled by varying the magnitude of maximum to average axial velocity $\left(u_{h} / \bar{u}\right)$, and the ratio of the height of the wave to the outer diameter of the pipe (h/Do), see Figure 6 . 


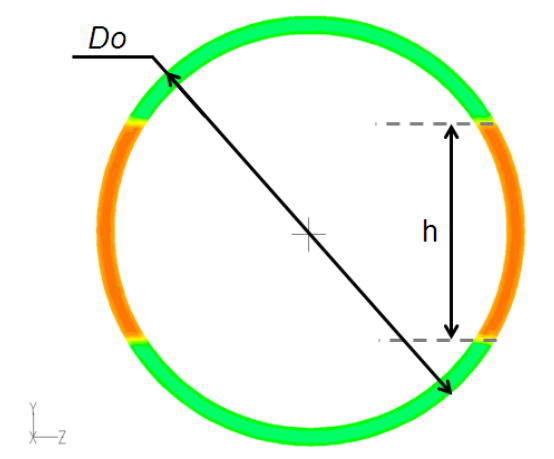

Figure 6: Square Distortion Inlet Profile Example (BC05)

The porous medium loss coefficient, $\mathrm{K}=\mathrm{K}_{\mathrm{x}}=\mathrm{K}_{\theta}$ (as $\theta_{\mathrm{k}}$ has been assumed to be unity) was calculated initially using the simplified form of Equation 11 and knowledge of the flow areas and vane angles. This resulted in an initial prediction using a porous media loss coefficient, $\mathrm{K}$, of $547 \mathrm{~m}^{-1}$. An additional solution was generated for the same model and mesh but with a zero loss coefficient. The solutions were compared on several planes upstream of the vane leading edge, example profiles are presented for the vane leading edge $(\mathrm{x} / \mathrm{L}=0.0)$ plane.

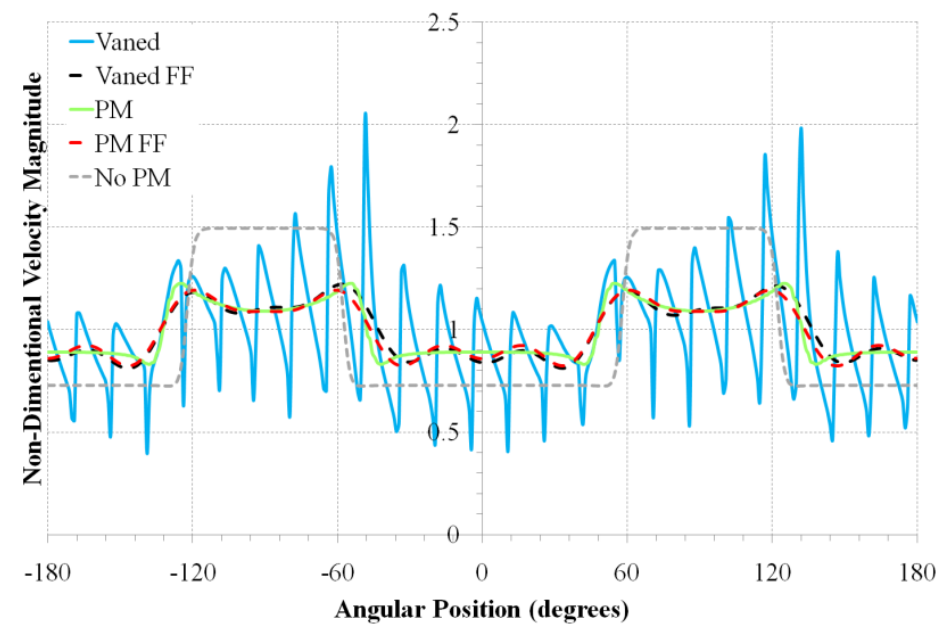

Figure 7: Velocity Profile at $x / L=0.0(B C 05)$

The vaned calculation shows the highly localised effects on the velocity field associated with individual swirl vanes. These perturbations vary between vanes as the incidence of the flow onto individual vanes varies with circumferential position. Such effects only extend a small distance upstream, as will be subsequently shown. It is impossible to capture the vane potential field effect with the PM method. However, it is the nonuniformity of the bulk average velocity field, as determined by the upstream flow field, and the interaction that occurs between the approaching flow and fuel injector which is of primary interest. 
To enable comparison between the PM and vaned solutions, a $10^{\text {th }}$ order Fourier fitted curve was created from the solution. The localised effects associated with the potential field of each vane were hence eliminated by virtue of the fact that the order of the fit was less than the vane count. The Fourier fitted curve and the two porous media approximations $\left(\mathrm{K}=547 \mathrm{~m}^{-1}\right.$ and $\left.0 \mathrm{~m}^{-1}\right)$ are presented in Figure 7 . It is immediately evident that the Fourier fitted approximation closely resembles the PM solution, although some subtle differences are apparent. For example, the vaned solution exhibits a slight asymmetric profile due to local incidence effects whereas the PM solution is symmetric. However, generally speaking the PM has captured the interaction that occurs with the upstream flow field which results in a more uniform flow at this axial location. In contrast the zero loss PM case has not captured any of this interaction, resulting in a vastly different and more non-uniform profile which is erroneous.

Comparisons can also be made at other axial locations upstream of the injector swirl vane location. This is quantified in terms of a simple error term based on the local differences in velocity relative to the vaned solution in which the complete flow field was modelled, i.e.

$$
E=\sum_{i=1}^{I}\left|\frac{C_{m i}}{\overline{C_{m}}}-\frac{C_{v i}}{\overline{C_{v}}}\right|
$$

Where: $\bar{C}$ is the area weighted average velocity magnitude at the axial plane being examined. Subscripts $v$ and $m$ refer to the vaned or porous solutions respectively. The index $i$ refers to the $i$-th circumferential point of which there are I in total. By plotting this error term as a function of axial position (Figure 8) it is possible to see the extent to which the PM is affecting the solution. When a PM is used the error is less than $1.2 \%$ up to 0.5 chord lengths from the vane leading edge position. This error increases rapidly close to the vane leading edge, as the velocity profile is affected by the potential field from the vanes. However, comparison with the Fourier fitted data indicates these potential flow field effects are only of significance within half a blade chord of the vanes. Comparisons can also be made with the exit boundary condition when the PM loss coefficients were set to zero. In this case the accuracy is poor with errors in velocity magnitude greater than $7.0 \%$ even 2 media lengths upstream of the vane leading edge. It is clear that, as anticipated, this solution does not accurately capture the interaction that occurs between the injector and approaching flow field. 


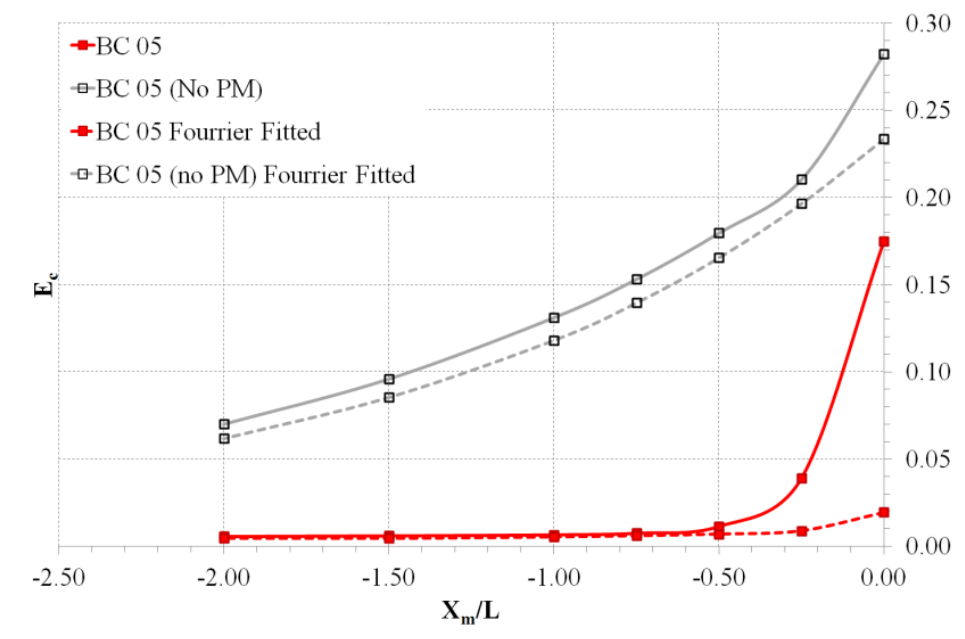

Figure 8: Upstream Comparison PM/ no PM (BC05)

\subsection{Sensitivity to Inlet Conditions}

In order to test the sensitivity of the porous media to the approach conditions the pipe geometry was used with 15 different boundary conditions (Table 2). These covered a range of inlet distortion magnitudes, patterns and variation of the bulk swirl angle (to indicate sensitivity to approach angle). For each boundary condition two solutions were computed: (i) a datum solution using the vaned geometry and (ii) a solution with the vanes replaced by a porous media with an appropriate loss coefficient, K (which was re-calculated from Equations 11 and 15). From each solution the error, E, between the vaned and porous media solutions was calculated at seven upstream planes (0-2 chord lengths). The maximum error (at $\mathrm{x} / \mathrm{L}=0$ ) is also listed in Table 2 for each inlet distortion pattern. The porous media performs well over the entire range of inlet conditions considered, the maximum error being consistently less than $2.5 \%$, and less than $0.5 \%$ beyond one chord length upstream.

\begin{tabular}{|l||l|l|l|l|l|}
\hline $\mathbf{B C}$ & $\boldsymbol{u}_{\boldsymbol{h}} / \overline{\boldsymbol{u}}$ & $\boldsymbol{h} / \boldsymbol{D}_{\boldsymbol{o}}$ & $\boldsymbol{v}_{\boldsymbol{\theta}}(\mathbf{m} / \mathbf{s})$ & $\boldsymbol{f}(\mathbf{H z})$ & $\mathbf{E}(\mathbf{x}=\mathbf{0})$ \\
\hline $\mathbf{1}$ & 1.1000 & 0.8 & 0 & Square & 0.008 \\
\hline $\mathbf{2}$ & 1.1000 & 0.5 & 0 & Square & 0.004 \\
\hline $\mathbf{3}$ & 1.1000 & 0.3 & 0 & Square & 0.004 \\
\hline $\mathbf{4}$ & 1.3000 & 0.5 & 0 & Square & 0.012 \\
\hline $\mathbf{5}$ & 1.5000 & 0.5 & 0 & Square & 0.020 \\
\hline $\mathbf{6}$ & 1.1000 & Sinusoid & 0 & 2 & 0.005 \\
\hline $\mathbf{7}$ & 1.1000 & Sinusoid & 0 & 4 & 0.007 \\
\hline $\mathbf{8}$ & 1.1000 & Sinusoid & 0 & 6 & 0.006 \\
\hline $\mathbf{9}$ & 1.3000 & Sinusoid & 0 & 4 & 0.016 \\
\hline $\mathbf{1 0}$ & 1.5000 & Sinusoid & 0 & 4 & 0.025 \\
\hline $\mathbf{1 1}$ & 1.4367 & 0.5 & 10 & Square & 0.015 \\
\hline $\mathbf{1 2}$ & 1.4845 & 0.5 & 5 & Square & 0.021 \\
\hline $\mathbf{1 3}$ & 1.4974 & 0.5 & 2 & Square & 0.020 \\
\hline $\mathbf{1 4}$ & 1.3541 & 0.5 & 15 & Square & 0.012 \\
\hline $\mathbf{1 5}$ & 1.2284 & 0.5 & 20 & Square & 0.009 \\
\hline
\end{tabular}

Table 2: Tested Inlet Boundary Conditions 


\subsection{Sensitivity to Terms in the K Equation}

In the calculations presented previously it was assumed that the porous loss coefficients were equal $\left(\theta_{\mathrm{k}}=1.0\right)$. To justify this assumption calculations were undertaken, again using BC05 as the inlet profile, over a range of $\theta_{\mathrm{k}}$ values. The velocity profiles at the porous medium inlet face $(\mathrm{x} / \mathrm{L}=0.0)$ were examined using the error function (E). The presented errors (Figure 9) justify the choice of value and suggest that losses are equally attributable to the axial and tangential velocity components (i.e. it is the total velocity that is of important rather than the individual components). It is likely however, that the optimum value of $\theta_{\mathrm{k}}$ will depend on the geometry of the vanes being modelled. An additional set of vanes were also tested and these gave an optimum $\theta_{\mathrm{k}}$ of around 1.4. Both vane sets were taken from real injector geometries and it is therefore reasonable to assume the value of $\theta_{\mathrm{k}}$ is in the range 0.9 to 1.4 . To attain the best possible accuracy the optimum value of $\theta_{\mathrm{k}}$ should be sought specifically for the geometry being investigated. It is also worthwhile noting that, although Figure 9 suggests that in the region of the optimum value the solution accuracy is highly dependent on the value of $\theta_{\mathrm{k}}$, the absolute error in the solution is small even when assuming a value of $\theta_{\mathrm{k}}$ of 3 or more. The PM solution always outperforms the non-porous case.

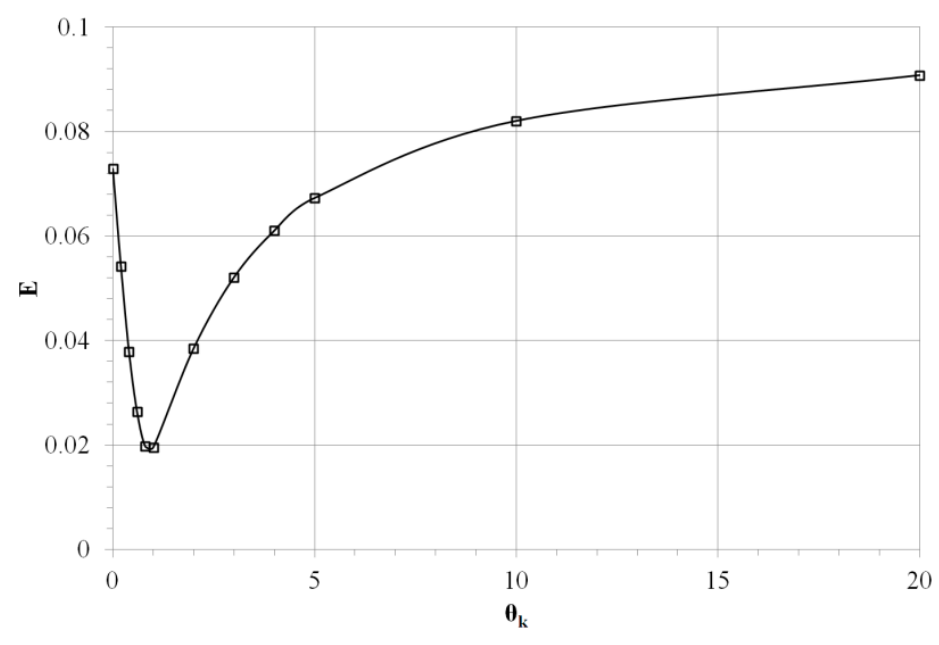

Figure 9: Variation of Error, $E$, as a Function of $\theta_{k}(x / L=0.0, B C 05)$

There are 7 other variables (excluding $\theta_{\mathrm{k}}$ ) to which values must be attributed in order to calculate $\mathrm{K}$ (Eqn. 11) as listed in Table 3 below. Some of these variables are known absolutely and may be measured for any particular geometry (e.g. the inlet and outlet areas of the passage). Some values can be approximated based on knowledge of the geometry (e.g. the exit angle, which is likely to be close to the swirl vane angle). The remaining values are flow dependent and must be assumed. It is the purpose of this section to identify the 
sensitivity of the solution to the values chosen. In order to do this, the pipe geometry will be utilised. It is likely that the actual sensitivity may have some dependency on the geometry being considered. However, the simplicity of the case allows the effect of the various terms to be investigated much more readily than in a full engine geometry.

\begin{tabular}{|l||c|l|l||}
\hline \multicolumn{1}{|c|}{ Term } & Symbol & \multicolumn{1}{c|}{ Comment } & \multicolumn{1}{c||}{ Value } \\
\hline \hline PM Length & $L$ & Defined by user & Measurable \\
\hline Geometric inlet Area & $A_{1}$ & Known & Measureable \\
\hline Geometric Outlet Area & $A_{2}$ & Known & Measureable \\
\hline BL blockage & $B$ & Flow dependant & 0.99 to 1 \\
\hline Inlet Angle & $\gamma_{1}$ & Flow dependant & 0 to $60^{\circ}$ \\
\hline Exit Angle & $\gamma_{2}$ & Approximately Known & swirl vane angle $+/-5^{\circ}$ \\
\hline Vane Total Pressure Loss & $\Delta P_{v} / q_{1}$ & Flow dependant & 0 to 1 \\
\hline \hline
\end{tabular}

Table 3: K Equation Variables and Approximate Values

The pipe flow case has the following geometric constants: $L=0.01 \mathrm{~m}, \mathrm{~A}_{1}=572 \mathrm{~mm}^{2}, \mathrm{~A}_{2}=476 \mathrm{~mm}^{2}, \gamma_{2}=$ $57^{\circ}$ (nominally). The variation in $\mathrm{K}$, given these constraints, and assuming that $\mathrm{B}=1.0$ is shown in Figure 10 . Note that these are analytical solutions, rather than CFD sought solutions, and as such it has been necessary to assume a value for $\gamma_{2}$ ' so that the gamma approximation may be used. It is noticeable from the figure that the value of $\mathrm{K}$ varies more significantly with a change in exit (swirl vane) angle than it does with the variation in vane total pressure loss parameter. This is because the vane total pressure loss is an additive term whereas the value of $\mathrm{K}$ depends on one over the square of the cosine of the outlet angle. For outlet angles approaching $90^{\circ}$ this term rapidly tends to infinity. Therefore, the heavy dependence on this term is unsurprising especially with such high swirl vane angles. Fortunately, the outlet angle of the flow is likely to follow quite closely the swirl vane geometric angle and may be reasonably estimated.

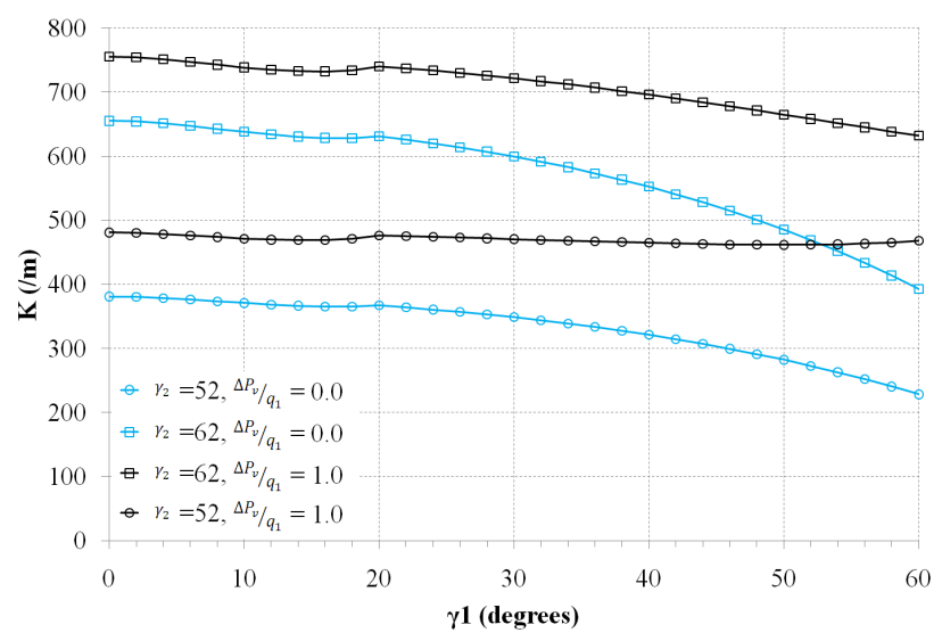

Figure 10: Variation in $\mathrm{K}$ as a Function of Inlet Angle, Outlet Angle and Total Pressure Loss 
For a specific boundary condition case (BC05) which is likely to be reasonably representative of a realistic geometry the effect of changing the assumed variables was considered. There are many variables so in order to cut down the amount of investigation two “corner points” were investigated: (i) $\mathrm{K}=381 \mathrm{~m}^{-1}$, which is equivalent to a $52^{\circ}$ exit angle and a zero total pressure loss vane cascade, and (ii) $\mathrm{K}=755 \mathrm{~m}^{-1}$, which represents a 62 degree outlet angle and a total pressure loss equivalent to the inlet dynamic head. These points represent the extremes of the envelope of $\mathrm{K}$ values. Running simulations with these values for $\mathrm{K}$ will allow an examination of the sensitivity of the velocity field to the value of $\mathrm{K}$.

Figure 11 presents the circumferential profiles of non-dimensionalised velocity magnitude for the datum (vaned) case and two porous media cases which represent the two extremes of the "K envelope". It may be seen that the velocity profile is reasonably insensitive to the value of $\mathrm{K}$ and both solutions provide reasonably accurate results. The expected value of $\mathrm{K}$ for this case, as detailed in Section 3, was $539 \mathrm{~m}^{-1}$ (after adjustment for $\gamma)$. Therefore the maximum and minimum $\mathrm{K}$ values represent a range of approximately $+/-40 \%$. However, the average absolute solution error at the leading edge location (the position of greatest error) is approximately $3.1 \%$ compared to the datum. When the expected value of $\mathrm{K}$ is utilised this error is reduced to $2.0 \%$. Therefore a $40 \%$ variation in the value of $\mathrm{K}$ represents an inaccuracy in velocity profile of around $1 \%$. Because the velocity profile is so insensitive to the value of $\mathrm{K}$ it is reasonable to use common or recommended value for the unknown quantities within the K equation. The most crucial component of this equation is the exit (swirl vane) angle and this may be estimated with a good degree of accuracy given knowledge of the vane geometry and typical flow behaviour. It is therefore possible to proceed with some confidence that the values obtained from initial investigations may be widely applicable and it is the recommendation of the authors that the following values be used, see Table 4.

\begin{tabular}{||l||c|l||}
\hline \multicolumn{1}{|c|}{ Term } & Symbol & \multicolumn{1}{c||}{ Value } \\
\hline \hline BL blockage & $B$ & 1.0 \\
\hline Inlet Angle & $\gamma_{1}$ & Calculate - iterative \\
\hline Exit Angle & $\gamma_{2}$ & Geometric swirl vane angle \\
\hline Vane Total Pressure Loss & $\Delta P_{v} / q_{1}$ & 0.6 \\
\hline
\end{tabular}

Table 4: Recommended Values for Unknown Terms of the K Equation 


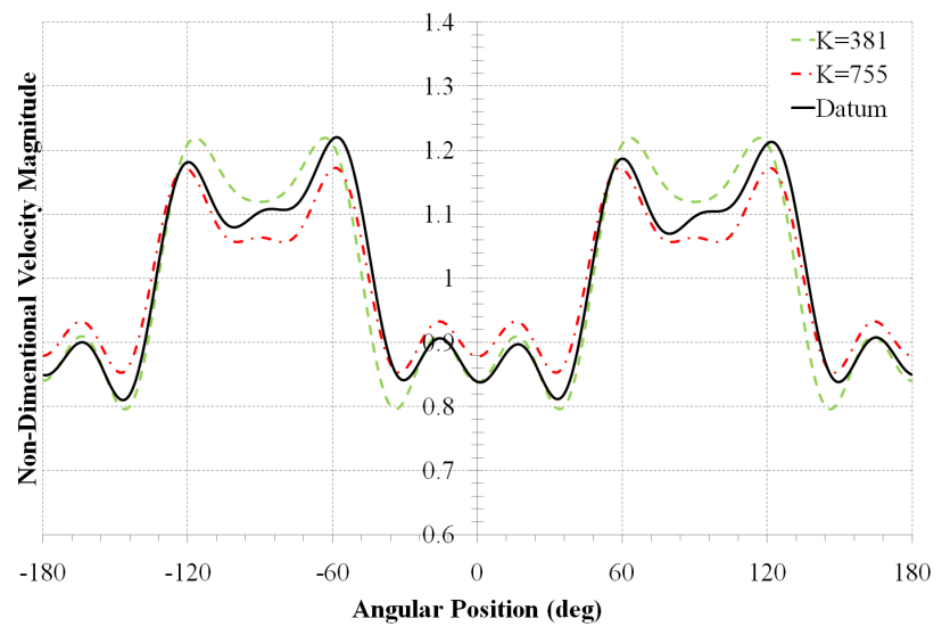

Figure 11: Variation in Velocity Profile with $\mathrm{K}(\mathrm{x} / \mathrm{L}=0.0, \mathrm{BC} 05)$

\subsection{Application to Engine Representative Combustion System Geometry}

The main aim of the current work was to establish the porous media methodology for modelling fuel injector swirl vanes in realistic gas turbine combustors thereby saving valuable time in the preliminary design process. The final stage in validating the methodology was therefore to apply it to a representative geometry for which a fully featured and complete CFD solution existed. The model extends from OGV exit, through the prediffuser and into the dump region whilst also including two annuli surrounding the flame tube. The annuli are truncated and are merely present to provide the correct mass flow distribution within the system, rather than to specifically investigate the annulus flows. The injector external geometry is common between both cases but the internal geometry and downstream flame tube depends on whether a fully featured or porous media approach is used. In the baseline case all the significant features of the injector and downstream combustor are modelled. However, in the porous case the vanes within each injector passage are replaced by porous media (starting at the leading edge position and extending approximately one chord downstream). Each injector passage has an individual exit boundary which may be used to adjust the mass flow split between the different injector passages. Example meshes (x-r plane) for the two cases are shown in Figure 12. Obviously for the full system model the mesh extended through each injector swirl vane passage and into the flame tube. As with the pipe geometry a calculation was also undertaken with "zero loss porous media” to enable the exact effect of the PM to be illustrated. 


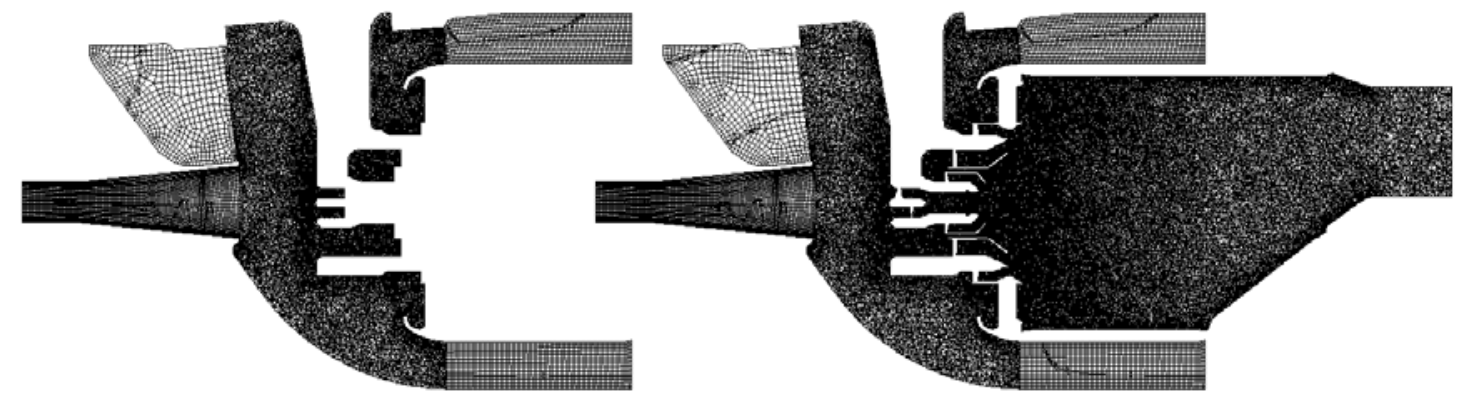

Figure 12: Centre-Planes of Domain With and Without PM Present

\subsection{CFD Methodology}

For the porous media case the vanes and downstream combustor geometry were removed and the domain re-meshed maintaining, where possible, the original mesh density and type. Three meshes were considered, as detailed in Table 5, covering a range of types and densities. However, comparison of the converged solutions suggested that similar results were obtained from all three. Mesh 3 was considered to be the most suitable for comparison since it represents the coarsest mesh and, therefore, gives the largest saving in computational effort. All future comparisons between the baseline datum case and the porous case are conducted using mesh 3.

Table 5 shows the vast reduction in computational effort achieved via the use of the PM method. The computational mesh is some $60 \%$ smaller than the fully featured geometry and reduces the computation time by almost 70\%. (On a single processor this is equivalent to reducing the computation time from 20 days to almost 5 days). Additionally, there is also a significant saving in the time and effort required to mesh the domain. The simplicity of the PM case allows the domain to be readily meshed whereas the fully featured geometry requires significantly more attention in order to generate a good quality mesh. Though this time saving is difficult to quantify it is significant and should not be overlooked. This is particularly true in the context of a new design or research project where many geometries may need to be investigated. It is also worthwhile to note the advantages of smaller solution domains with regard to data storage and post processing which are made easier by a reduction of cell count.

Throughout all the numerical investigations, a consistent methodology was adopted for all other modelling aspects. For example in each case a RANS methodology was employed with turbulence closure achieved using a Realisable k- $\varepsilon$ turbulence model in conjunction with standard wall functions. The choice of turbulence model will obviously influence the solution but the purpose of the current investigation is to compare the effect of the porous media to a fully featured geometry. Consequently, the influence of the choice of turbulence model was 
not considered. For simplicity, all calculations were undertaken under isothermal (ISA standard day) conditions since any change in pressure loss associated with heat release has a minimal impact on the combustor external aerodynamics. Further the flow was assumed to be incompressible.

\begin{tabular}{||l||c|c|c|c||}
\hline & Full Model & Mesh 1 & Mesh 2 & Mesh 3 \\
\hline \hline Pre-Diffuser & 0.46 & 0.46 & 0.46 & 0.46 \\
\hline Inner Annulus & 0.07 & 0.07 & 0.07 & 0.07 \\
\hline Outer Annulus & 0.12 & 0.12 & 0.12 & 0.12 \\
\hline Dump Cavity & 2.03 & 1.86 & 2.04 & 2.00 \\
\hline Flame Tube & 3.49 & 0.00 & 0.00 & 0.00 \\
\hline Injector & 1.47 & 1.18 & 2.29 & 0.50 \\
\hline Of which is PM & 0.00 & 0.30 & 1.44 & 0.16 \\
\hline \hline Total Cell Count & $\mathbf{7 . 6 5}$ & $\mathbf{3 . 6 9}$ & $\mathbf{4 . 9 8}$ & $\mathbf{3 . 1 5}$ \\
\hline \% of Full Model & $\mathbf{1 0 0 . 0}$ & $\mathbf{4 8 . 3}$ & $\mathbf{6 5 . 2}$ & $\mathbf{4 1 . 2}$ \\
\hline $\begin{array}{l}\text { Simulation Time } \\
\text { (\% of Full Model) }\end{array}$ & $\mathbf{1 0 0 . 0}$ & $\mathbf{5 3 . 5}$ & $\mathbf{8 7 . 9}$ & $\mathbf{3 0 . 5}$ \\
\hline
\end{tabular}

Table 5: Mesh Details (cell count in millions)

The inlet boundary conditions were provided by a fixed velocity assumption since the velocity profile at OGV exit was known. The domain was reduced to a single injector sector with periodic end wall and simplified such that there were only 7 exits; the injector (containing 5 sub passages) and the inner and outer annuli. Each exit condition was modelled as a fixed mass flow outlet with approximately $70 \%$ of the total inlet mass flow passing through the injector. The various sub passage flow splits were set from knowledge of the ideal injector effective area distributions. In the fully featured case the injector passage outlet planes were replaced by a single exit plane at turbine nozzle guide vane inlet.

\subsection{Near Field Injector Flow Conditions}

Although some differences between the fully featured model and PM case are apparent in the far field region it is the flow field being presented to the fuel injector that is of most interest here (i.e. the injector 'near field' region). There are several discrete vane passages within the injector geometry but, in the interests of brevity, attention will be limited to only two passages. The one furthest from the injector centreline (i.e. the outer most swirler) will be referred to as Passage 1. The approach condition to this passage is particularly challenging, since the passage radii extends beyond the pre-diffuser exit height. Passage 2 is located further towards the centreline of the injector and sits entirely within the footprint of the upstream diffuser. These two passages accept the majority of the injector mass flow and thus demand the greatest attention.

To compare the near field accuracy of the porous medium method an examination of the area weighted, radially averaged, circumferential profile of velocity magnitude has been used. These profiles are taken on the 
PM inlet planes, which are coincident with the location of the swirl vane leading edge for the fully featured case. Profiles have been Fourrier fitted to a tenth order and are consistent with the methodology described in Section 3. In this way any localised effects caused by the pressure field associated with individual swirl vanes are removed. Any flow field variations reflect the large scale spatial variations that can be attributed to the external flow field and the way fluid is being supplied to the injector.

Figure 13 shows the velocity profiles for Passage 1 with an angular position of $0^{\circ}$ corresponding to the injector top dead centre. It is clear from the figure that use of the porous medium significantly improves the accuracy of the solution relative to the case with no porous media; the profile closely matching of the baseline. The average error in velocity magnitude is less than $4 \%$ when modelling with a porous medium whereas without the PM the error is in excess of 35\%. Also without the porous medium the flow distribution is incorrect and the majority of the flow is located towards the $90^{\circ}$ and $270^{\circ}$ positions giving rise to large velocities in these regions. This is a direct result of the pre-diffuser efflux imprinting itself onto the Passage 1 inlet face. However in reality and in the porous media case the flow is redistributed.

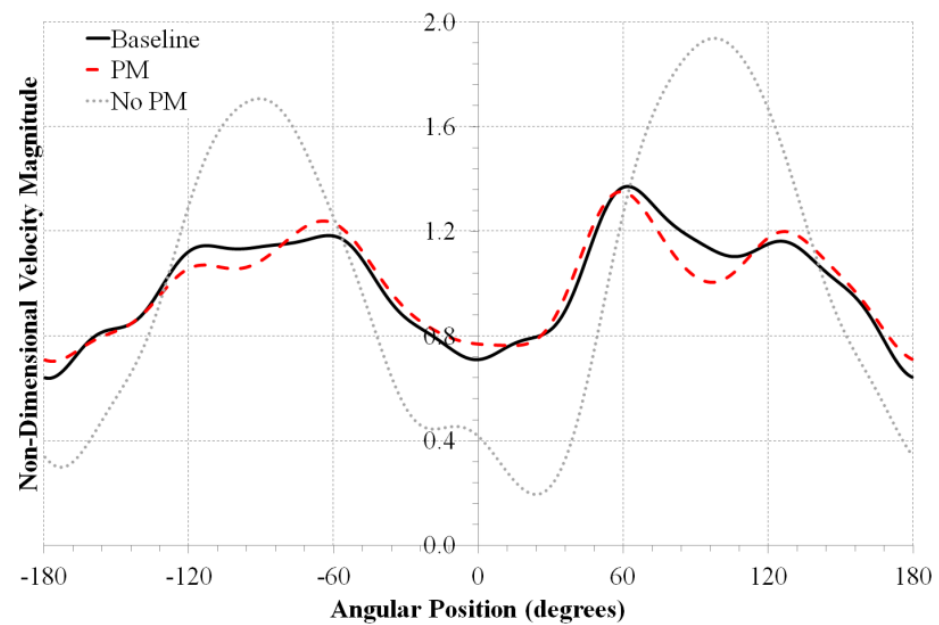

Figure 13: Passage 1 Circumferential Velocity Profile $(x / L=0.0)$

Whilst the use of porous media gives a flow distribution comparable with the baseline, some differences are apparent. For example, velocities are slightly under predicted at around $-112^{\circ}$ and $+90^{\circ}$ and slightly over prediction at $0^{\circ}$ and $\pm 180^{\circ}$. These errors come as a result of local flow incidence effects. The distorted profile presented to the Passage 1 inlet face has an array of flow angles ranging from approximately $\pm 60^{\circ}$. At extremely high angles of incidence the swirl vanes are liable to stall resulting in higher loss and a reduced flow rate. This will then lead to increased velocities in regions where the approach angles are small. The PM model would not be able to capture such extreme effects accurately. 
Figure 14 shows the circumferential velocity distribution for Passage 2 at the PM inlet face. The porous, non-porous and baseline cases are all plotted. Passage 2 appears to show less difference between the porous and non-porous cases although it is apparent that the porous solution better represents the baseline distribution. The average error in non-dimensional velocity is less than $3.0 \%$ in the porous media case. The non-porous case has an average error of approximately $6.0 \%$. In this case the flow is influenced by an upstream feed arm (at $0^{\circ}$ ) through which fuel is supplied to the central region of the injector. This is responsible for the large velocity deficit seen in this region and is located less than one chord length upstream of the swirl vanes. Hence the local potential flow field associated with individual swirl vanes interact with the fuel feed arm. This also accounts for the asymmetry of the velocity deficit in this region observed in the baseline calculation. This mechanism, though, cannot be captured by the porous media and so some differences are apparent. Nevertheless it is thought that the porous media generally gives a reasonably good indication of flow uniformity.

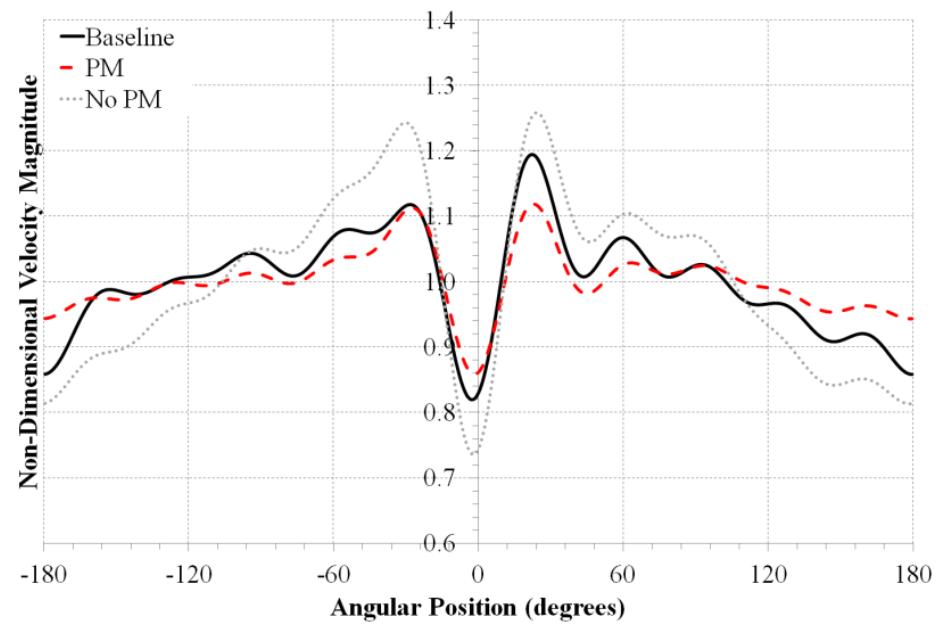

Figure 14: Passage 2 Circumferential Velocity Profile $(x / L=0.0)$

\subsection{Concluding Remarks}

The importance of capturing the effects of the injector swirl vanes and downstream combustor flow field on the upstream flow field external to the flame tube has been demonstrated. This is thought to be of particular significance for future aero style gas turbine combustion systems in which the uniformity of flow presented to the injector can significantly impact emissions performance. To avoid calculating the flame tube internal and external flow fields an alternative approach has been presented in which the swirl vanes and downstream combustor flow field are replaced with porous media. The advantage of this approach is that it provides a 
significant reduction in simulation time whilst still enabling the quality of the flow (pressure loss, flow uniformity) being presented to the injector swirl vanes to be evaluated to a good first order engineering approximation. The methodology has been shown to be robust, practical and can be achieved through suitable knowledge of the injector geometry along with the suitable values outlined in this report. Hence this makes the technique particularly useful during the initial design phase of a project. Once the geometry has been refined a fully featured calculation may be run and used to define the final geometry safe in the knowledge that the initial predictions generated using porous media are accurate enough to capture the gross fluid behaviour.

\section{References}

[1] Fishenden, C. R. and Stevens, S. J., 1977, "Performance of Annular Combustor Dump Diffusers", Journal of Aircraft, Vol. 14, pp. 60-67.

[2] Carrotte, J. F., Bailey, D. W. and Frodsham, C. W., 1995, "Detailed Measurements on a Modern Combustor Dump Diffuser System”, ASME Journal of Engineering for Gas Turbines and Power, Vol. 117, pp.678-685.

[3] Walker, A. D., Carrotte, J. F. and McGuirk, J. J., 2008, “Compressor/Diffuser/Combustor Aerodynamic Interactions in Lean Module Combustors”, ASME Journal of Engineering for Gas Turbines and Power, Vol. 130(1), pp. 011504-1-08.

[4] Barker, A. G. and Carrotte, J. F., 2002, "Compressor Exit Conditions and their Impact on Flame Tube Injector Flows”, ASME Journal of Engineering for Gas Turbines and Power, Vol. 124(1), pp. 10-19.

[5] Lefebvre, A. H., 1999, “Gas Turbine Combustion”, $2^{\text {nd }}$ Edition, Taylor and Francis.

[6] Lazik, W., Doerr, T. and Bake, S., 2008, “Development of Lean-Burn Low-NO ${ }_{x}$ Combustion Technology at Rolls-Royce Deutschland”, ASME Paper GT2008-51115.

[7] Walker, A. D., 2002, "Experimental and Computational Study of Hybrid Diffusers for Gas Turbine Combustors”, PhD Thesis, Dept. Aeronautical and Automotive Engineering, Loughborough University. 